\title{
Requisitos e critérios para projetos habitacionais: em busca de um desempenho ampliado
}

\author{
CASTRO, Maria Luiza Almeida Cunha de ${ }^{1}$ \\ LOURA, Rejane Magiag ${ }^{2}$ \\ 1Escola de Arquitetura, Universidade Federal de Minas Gerais, Belo Horizonte, Brasil. luizadecastro@ufmg.br \\ 2 Escola de Arquitetura, Universidade Federal de Minas Gerais, Belo Horizonte, Brasil. magiagloura@yahoo.com
}

\section{Resumo}

No Brasil, a entrada em vigor da norma NBR 15575, em 2013, traz garantias para a qualidade de edificações habitacionais ao estabelecer requisitos e critérios ligados, principalmente, às propriedades físicas de seus sistemas tecnológicos. Entretanto, a norma define unicamente um desempenho mínimo e de escopo limitado, que não contempla questões cruciais de uma forma mais abrangente - como a importância da interação entre a concepção do projeto e a escolha dos sistemas tecnológicos, a avaliação das respostas humanas ou a contribuição das escolhas para a sustentabilidade ou o desenvolvimento. Este artigo tem como objetivo colocar em pauta a necessidade de articulação dos projetistas, construtores, fornecedores e outros agentes tais como instituições e organismos reguladores em torno desta questão: a organização desta discussão trará subsídios para um aprendizado conjunto sobre os requisitos/atributos importantes a serem priorizados nas tomadas de decisão de projeto, no que diz respeito à busca de desempenho na habitação multifamiliar.

Palavras-Chave: habitação multifamiliar; análise de desempenho; análise multicritério; sustentabilidade; controle de qualidade.

\begin{abstract}
Effective in Brazil since 2013, ABNT Standard 15575 brings guarantees to the quality of multifamily housing, as it establishes requirements and criteria related primarily to the physical properties of the building's technological systems. However, the standard defines only minimum requirements and a performance of limited scope, which does not address crucial issues in a more comprehensive way - such as importance of the interaction between the conception of the project and the choice of technological systems, the assessment of human responses or the contribution to various aspects of sustainability or to development. This paper seeks to highlight the need of coordinating architects, builders, suppliers and other agents such as institutions and regulatory agencies involved in the construction industry around this issue: the organization of this discussion will bring subsidies for a learning process concerning requirements/attributes to be prioritized in decision-making, allowing for a performance improvement in multifamily housing.
\end{abstract}

Key-Words: multifamily housing, performance analysis; Multi-Criteria Decision Analysis; sustainability; quality control. 


\section{Introdução}

No Brasil, a entrada em vigor da norma NBR 15575, em 2013, representou uma conquista decisiva em direção à busca de garantias de qualidade para as edificações de habitação multifamiliar em sua fase de uso, impondo uma análise de desempenho: esta norma estabelece requisitos que visam suprir necessidades do cliente/usuário, determinando sua adequação a partir de critérios ligados, principalmente, às propriedades físicas dos sistemas tecnológicos que compõem a edificação.

Esta avaliação de desempenho se coloca em contraposição à análise tradicional, baseada em considerações de cunho prescritivo. Ela estabelece padrões a partir do comportamento da edificação, que é resultado de diversos fatores concomitantemente: a análise está focada na disponibilização de uma condição ideal para o uso de cada sistema e não unicamente na conformidade dos componentes do mesmo isoladamente.

Entretanto, a norma define unicamente uma performance mínima e de escopo limitado. De uma parte, ela deixa espaço para uma escolha entre a condição normal de uso e um estado limite (SILVA, 1996), proporcionando muitas vezes apenas um "desempenho mínimo aceitável que leva a soluções à beira do inaceitável” (MANN; BENDER, 1972). De outra parte, os critérios passíveis de aferição laboratorial têm prioridade, mas "a interação destes com os aspectos relacionados à concepção de projeto são pouco explorados pela investigação em pesquisas ou pelos esforços de aplicação prática" (SILVA, 1996, p 130). Adicionalmente, algumas questões só são contempladas de forma indireta, como por exemplo, aquelas avaliadas por meio de respostas humanas, as referentes à gestão do empreendimento ou as que dizem respeito ao impacto da edificação na economia local e na sustentabilidade de uma forma mais abrangente.

O foco da norma nas questões tecnológicas retrata, então, uma falta de integração sistêmica recorrente nos processos de tomada de decisão, os quais, de acordo com UNEP (1992), tendem a separar fatores econômicos, sociais e ambientais, nos níveis de políticas, planejamento e gestão. Esta estanqueidade é derivada de uma visão que tem suas origens em âmbitos da macro escala e está claramente presente no planejamento e gestão de empreendimentos ligados à construção da habitação multifamiliar no Brasil. A falta de integração impede que haja uma abordagem ampliada ou a compatibilização das muitas informações e demandas e, para suprir estas deficiências, novas formas de diálogo entre os agentes de cada esfera devem ser configuradas (UNEP, 1992).

$\mathrm{Na}$ construção, que envolve a intervenção de agentes autônomos e com motivação própria, a insuficiência no compartilhamento de valores é especialmente crítica: dados, expertise e controle são fragmentados e distribuídos (UTOMO, IDRUS, 2011), inviabilizando um conhecimento aprofundado do todo. As decisões são tomadas de acordo com pontos de vista unilaterais privilegiando em geral a ótica do empresário, voltada para a questão econômico-financeira de sua empresa. Perspectivas mais amplas, que se traduziriam no atendimento de necessidades dos futuros usuários e da sociedade em geral, são relegadas ao segundo plano para priorizar os indicadores de viabilidade do projeto para a empresa.

O problema crucial que se coloca, portanto, é mais amplo do que a preocupação que a norma expressa com a conformidade dos sistemas tecnológicos em fase de uso, e é anterior a qualquer análise do desempenho - ele diz respeito à própria escolha dos requisitos e critérios a serem colocados em avaliação, uma vez que estes devem refletir as reais necessidades dos envolvidos, inclusive da sociedade como um todo.

A pesquisa realizada teve caráter exploratório e procurou, então, examinar a literatura no que se refere à importância da definição de atributos para as tomadas de decisão de projeto. Foram levantados trabalhos que avaliam as possibilidades de lidar com as demandas e opiniões divergentes que surgem nas discussões, a partir das teorias de suporte à decisão. A investigação se justifica com base em Roy (1998), que propõe, para pesquisas "multicritério", a prospecção de condições e meios nos quais as decisões possam se basear, à luz do que se acredita ser o mais adequado, contribuindo, assim, para a construção, estabelecimento e discussão de convicções. De acordo com Van Der Heijden (2004), trabalhos desta natureza partem de uma perspectiva 
que não visa especificamente a tomada de decisão em si, mas a organização dos processos de decisão.

\section{Requisitos e critérios: uma escolha anterior à definição de desempenho}

A Norma Brasileira para Avaliação de Desempenho, NBR 15575:2013, é a referência para a avaliação dos sistemas tecnológicos nas edificações habitacionais e tem por base uma lista de necessidades dos usuários para a fase de uso das edificações: (i) segurança, (segurança estrutural, segurança contra fogo e segurança no uso e na operação) (ii) habitabilidade (estanqueidade, desempenho térmico, desempenho acústico, desempenho lumínico, saúde, higiene e qualidade do ar, funcionalidade e acessibilidade, conforto tátil e antropodinâmico) e (iii) sustentabilidade (durabilidade, manutenabilidade e impacto ambiental).

Estas exigências foram definidas inicialmente por uma comissão de trabalho do Conseil International du Bâtiment - CIB (CIB W 45), criada em 1960 e mais tarde consolidadas na ISO 6241:1984 (SILVA, 1996). Elas foram estabelecidas e os critérios para seu cumprimento, estruturados, dentro de uma articulação nos moldes do tema que é conhecido como Teoria da decisão ou ainda "processo decisório", "metodologias de auxílio à tomada de decisão", ou "auxílio à decisão" (PICANÇO, 199?). Sob esta perspectiva, o processo decisório se faz a partir da definição de atributos ou requisitos de uma performance esperada, que são traduzidos em critérios mensuráveis, a fim de que possam ser objetivamente determinados, servindo como referência para a avaliação de cada processo ou sistema.

Os critérios incluem parâmetros que definem as condições para que os objetivos desejados ou necessários de uma escolha tecnológica sejam alcançados. Os parâmetros, por sua vez, refletem ou caracterizam a medida na qual um critério é atendido dentro das condições específicas de exposição de cada empreendimento.

A inclusão de requisitos relativos à sustentabilidade aproxima significativamente a norma brasileira das atuais discussões internacionais, especialmente no que diz respeito à durabilidade. Até a entrada em vigor desta norma, a maior parte dos trabalhos voltados para estes temas estava restrita à academia. Ela agora impõe a aplicação da análise em casos reais, o que tende a ser muito significativo quando se trata da qualidade dos produtos e avanço das pesquisas científicas na área. A NBR 15.575:2013 trouxe para o mercado brasileiro uma ampliação dos objetivos dos empreendimentos, antes focados unicamente no lucro. Tal ampliação visa contemplar também necessidades durante o uso, procurando-se, desta maneira, um equilíbrio entre a performance e o custo para atingi-la.

Embora recente no Brasil, tal abordagem já está consolidada em países como França, Inglaterra, Estados Unidos, Austrália, entre outros. O conceito de desempenho foi introduzido pelo pesquisador inglês F. M. Lea, do Building Research Establishment, no segundo congresso do CIB, realizado em 1962 (SILVA, 1996). A avaliação de desempenho, entretanto, depende do sistema de expectativas quanto aos campos em que o mesmo deve ser exigido, questão que se expressa na escolha dos requisitos, critérios e parâmetros adotados para mensurá-lo. Em sua forma mais abrangente os parâmetros dependem não só de fatores físicos - tais como clima, relevo, situação - mas também de fatores econômicos - infraestrutura, recursos capacidade econômica - e fatores sociais, culturais e políticos - tradições, demandas, organização social e política (GROSS, 1996).

Os limites a serem contemplados na avaliação de cada sistema têm sido alvo de questionamentos desde os primeiros esboços de formatação das metodologias e estruturação de instrumentos de regulação. Assim, uma das primeiras articulações efetivas em torno da questão do desempenho ocorreu a partir de 1969 nos Estados Unidos, sob a forma de uma operação, conhecida como “Breakthrough", coordenada pelo Departamento de Habitação e Desenvolvimento urbano (Department of Housing and Urban Development BUD). O programa consistiu na implantação de normas e diretrizes baseadas no desempenho em vários estados americanos, permeando um ambiente de estímulo à inovação tecnológica, com o objetivo de implementar a melhoria nos programas habitacionais de baixa renda e encorajar a inovação em sistemas tecnológicos (FINGER, 1972). A importância da definição de critérios de projeto bem 
determinados foi colocada em pauta por uma autocrítica ao programa: de acordo com o relatório feito ao governo em 1976, a experiência com a operação demonstrou que programas futuros, além de avaliar questões técnicas e mercadológicas, deveriam envolver um "trabalho preliminar cuidadoso para desenvolver critérios" (STAATS, 1976, p. 1).

A estruturação de requisitos e critérios continuou a ocupar posição de destaque enquanto outras frentes de estudo e divulgação do conceito foram adquirindo maior amplitude. A importância que a discussão assumiu fica evidente a partir do envolvimento de instituições como a ASTM e a RILEM - que, em 1972, organizaram conjuntamente com o CIB na Filadélfia o primeiro Simpósio Building for People: Behavioral Research Approaches and Directions. Nesta ocasião foi apresentado o estado da arte das pesquisas em curso, por meio de 82 trabalhos selecionados entre pesquisadores de várias partes do mundo, que mostraram os esforços para desenvolvimento da nova abordagem e trataram dos muitos aspectos da análise de desempenho (FOSTER, 1972). Pelo menos uma dezena dos trabalhos apresentados abordou mais especificamente a estruturação dos requisitos e critérios, bem como os tipos de informação necessária para tal - entre outros, Pena; Focke (1972); Cronnberg et al (1972a; 1972b); Lemer; Moavenzadeh (1972).

Ao longo das décadas seguintes, a comissão W60 do CIB continuou dando substância à investigação sobre o assunto (SILVA, 1996) e outras instituições, como a Unidade de Pesquisa de Desempenho na Construção da Universidade de Strathclyde (MARTHUR; McGEORGE, 2006), trouxeram definitivamente a questão da performance em uso para o centro das atenções (VAN DER HEIJDEN, 2004).

Uma experiência pioneira ocorrida na Bélgica, em 1979, em um concurso de projetos de Arquitetura organizado em Liége (D'HAVÉ, 1981 apud SILVA, 1996) colocou em destaque as possibilidades trazidas pela inclusão de requisitos de desempenho focados no desenvolvimento. As propostas apresentadas pelos arquitetos concorrentes visando à construção de duas mil e quinhentas unidades habitacionais foram julgadas com base na verificação de questões tais como: o custo global ao longo de um período de vinte e cinco anos de uso; valores arquitetônicos; grau de industrialização do projeto; impacto do projeto para o desenvolvimento econômico da região. Nota-se, aqui, a ênfase na consideração das necessidades da sociedade de uma forma mais ampla.

A partir de então, questões referentes aos custos em uso foram sendo sistematicamente incorporadas às metodologias, contemplando aspectos como a taxa de degradação, deterioração da capacidade de prover os serviços - serviceability - e confiabilidade, gastos e frequência de manutenção e/ou reposição, consumo de energia e outros recursos (SILVA, 1996).

Hoje, a assimilação da mudança de paradigma introduzida pela noção do desempenho continua em curso - com grau de adiantamento proporcional ao estágio de industrialização da construção em cada país. A ênfase na importância da definição de requisitos que expressem uma simplificação representativa de cada realidade complexa é recorrente e tem merecido a atenção de pesquisadores tais como Ugwu; Haupt, (2007); Pasquire et al. (2005), Chen et al, (2010); Yunus; Yang (2012) os quais, em países diferentes realizaram pesquisas voltadas para a discussão de requisitos e critérios a partir da percepção dos vários stakeholders.

Este breve histórico ressalta o aumento do rigor dos critérios e ampliação dos parâmetros de análise, bem como o caráter permanente de busca por melhoria dos procedimentos para avaliação de desempenho

Em face deste contexto internacional, destaca-se a necessidade de atenção à situação brasileira, uma vez que as normas técnicas desenvolvidas pela Associação Brasileira de Normas Técnicas (ABNT), de modo geral, não são submetidas a uma revisão com regularidade periódica. Tal regularidade permitiria a atualização da abordagem de desempenho das edificações frente às possibilidades de melhorias disponíveis no mercado. Além disso, proporcionaria a criação e manutenção de fórum permanente para fomento desta discussão, elemento essencial para que se observe no país a mesma trajetória de ampliação da abordagem do desempenho nas edificações, vista nos países desenvolvidos. 


\section{Tendências emergentes em termos de requisitos}

Apontar as tendências emergentes em relação aos requisitos é uma forma de apontar os possíveis caminhos que a abordagem de desempenho brasileira irá trilhar. Os diversos recortes propostos pelos autores permitem uma visão de diferentes extratos da realidade e, quando se observa a recorrência da indicação de determinados requisitos enquanto preocupação prioritária de atores do setor, este é um indício do surgimento de novos padrões no sistema de expectativas da sociedade.

As pesquisas de campo realizadas mais recentemente mostram o aprofundamento das abordagens, que evoluíram no sentido de um maior cuidado com o bem-estar geral e o meio ambiente, em sintonia com as tendências sociais emergentes: Ugwu e Haupt (2007) propuseram na África do Sul categorias de indicadores de sustentabilidade a serem aplicados em um modelo de decisão e avaliação de sustentabilidade para projetos de infraestrutura em países em desenvolvimento: em sua pesquisa, eles submeteram uma seleção pré-definida de indicadores a um total de quarenta e nove participantes - atuantes na construção, incluindo as categorias - economia; meio ambiente, sociedade, reutilização; saúde e segurança. Os respondentes da pesquisa escolheram indicadores que consideravam importantes para sua subsequente inclusão em uma análise multicritério.

Pasquire et al. (2007), por sua vez, elaboraram um kit para ajudar a escolha entre os métodos tradicionais e os métodos pré-fabricados de construção, a partir da coleta de dados em empresas, consultores e clientes na Grã-Bretanha ao longo de três anos; um estudo piloto foi realizado com trinta organizações e mais de cem kits foram distribuídos no país e, em menor escala, em outros países da Europa e Estados Unidos. O kit proposto incluiu uma tabela comparativa com requisitos relativos a custo; tempo; qualidade; saúde e segurança; sustentabilidade e questões construtivas. Cada fator está inter-relacionado com os demais e para cada um destes atributos foram propostos critérios de avaliação.

Chen et al, (2010) colocaram o foco mais marcadamente sobre a escolha dos requisitos e critérios propriamente ditos: a partir de uma pesquisa sobre a percepção de clientes, empreiteiros, engenheiros, e fabricantes de pré-fabricados de concreto nos USA (com 95 questionários válidos), eles agruparam os requisitos em sete dimensões, incluindo fatores econômicos (por exemplo, tempo de construção, custos ao longo do ciclo de vida do edifício; construtibilidade, durabilidade, integração de cadeias de fornecedores; qualidade; custos materiais e custos de mão de obra, etc); fatores sociais (impacto na saúde dos ocupantes disponibilidade de mão de obra, saúde e segurança dos trabalhadores; impacto no mercado de trabalho; e impacto na comunidade; impacto no trânsito) e o fator ambiental (impacto ambiental, intensidade energética, intensidade material; desperdício, poluição gerada, consumo de água, etc).

A pesquisa realizada por Yunus e Yang (2012) na Malásia também teve como preocupação principal a identificação de requisitos relevantes para os diversos atores ao longo do ciclo de construção, por meio de questionários aplicados a diferentes agentes da construção. Eles identificaram três fases: a préconstrução, construção e pós-construção; as questões relevantes detectadas nesta pesquisa incluiram, na fase de pré-construção: a legislação, diretrizes de controle de projetos, sistema de compras, padronização, custos de material, produção, conhecimentos e habilidades, material consumo e geração de resíduos; na fase de construção, a disponibilidade de mão de obra, custo do trabalho, defeitos e danos, tempo de construção e condições de trabalho; na fase de pós-construção, a durabilidade, custo de manutenção e operação, uso, eficiência e eliminação de resíduos. Estas questões foram, então, agrupadas sob os temas: valor econômico, desempenho ecológico, eqüidade social e cultural, qualidade técnica, e implementação e execução.

O ponto em comum entre estas pesquisas foi o foco mais marcadamente sobre o estágio que precede a tomada de decisão: a escolha dos requisitos e critérios, que revela a visão do mundo dos agentes envolvidos. As metodologias envolveram, em cada caso o questionamento de diversos interventores na construção - incluindo arquitetos, engenheiros, empreiteiros, contratantes do setor público e gerentes de projeto - e outros questionamentos sobre 
os indicadores que cada um considerava importante para sua subsequente inclusão em uma análise multicritério.

$\mathrm{Na}$ maioria das pesquisas, os resultados foram extraidos e incorporados a uma listagem de referências para melhores práticas, usada no suporte a processos de tomada de decisão. (YUNUS; YANG, 2012, UGWU; HAUPT, 2007, CHEN et al., 2010) e no caso de Pasquire et al. (2005), os requisitos e critérios definidos foram aplicados a um estudo piloto, realizado com trinta organizações ao longo de três anos, no qual se procurou estabelecer uma comparação com requisitos relativos a custo; tempo; qualidade; saúde e segurança; sustentabilidade e questões construtivas. Um levantamento sobre a aplicação prática da avaliação revelou a dificuldade dos agentes envolvidos em coletar dados que pudessem quantificar os requisitos, mas destacou a tomada de consciência da importância em mudar o foco para questões que fossem além daquelas tratadas tradicionalmente nas tomadas de decisão. Foi constatada, ainda, a necessidade de mudar os processos de gestão de informação nas obras, para obter informações efetivamente relevantes para a tomada de decisão.

Uma análise destas pesquisas destaca, por um lado, a consolidação do tema sustentabilidade, em todas suas nuances, e por outro, o aprendizado empírico.

Assim, as principais preocupações que emergem estão ligadas à sustentabilidade: a) de cunho ambiental, expressa pela preocupação com diversos fatores ao longo do ciclo de vida contemplados ou não pelas normas voltadas pelo custo em uso, incluindo geração de resíduos, desperdício, poluição; a durabilidade, reutilização, intensidade energética, intensidade material; custo de manutenção e operação, uso, eficiência o consumo de água, e eliminação de resíduos, etc; b) de cunho social - mostrando-se em um estágio de expectativas mais avançado do que as exigências da norma, ao estabelecer uma preocupação com as condições de trabalho saúde e segurança dos trabalhadores, além do impacto na saúde dos ocupantes; c) de cunho econômico, no nível da sociedade, incluindo o questionamento sobre o impacto no mercado de trabalho; na comunidade ou no trânsito, por exemplo.
Como pode ser constatado, esta visão vai bem além da proposta atual da NBR. 15575:2013 e envolve em vários níveis as respostas humanas às condições criadas. A abordagem atual está focada principalmente na durabilidade dos sistemas construtivos analisados, com base na norma ISO 15686/2000 "Planejamentos de vida útil de edifícios e bens construídos", ou seja, limitada à questão ambiental. Porém mesmo estando limitada a um aspecto da sustentabilidade é preciso salientar que os construtores, fabricantes e projetistas brasileiros não estão preparados para seguir os métodos de determinação de vida útil de projeto, conforme determina a NBR15575:2013 (BERNARDES et al, 2014). O arcabouço de dados disponível fica muito aquém do necessário para se chegar a resultados confiáveis. Esse fato leva a questionamentos a respeito da pertinência do processo de construção da NBR15575:2013.

Voltando às pesquisas, observa-se também a consciência da influência marcante que o projeto exerce na produção e do fato de que o impacto das decisões nos custos é máximo durante a elaboração do projeto, momento em que os gastos são menores, tal como já anteriormente constatado por Barrie e Paulson, (1992 apud HYUN, 1997). O aporte de conhecimento trazido pelo construtor, que já deve estar envolvido com o projeto desde o seu desenvolvimento é, portanto, fundamental para o bom andamento do trabalho de todas as partes envolvidas.

Do ponto de vista da relação com a prática, estas pesquisas representam um passo importante em direção a um aprendizado estratégico, uma vez que permitem o reconhecimento de padrões de comportamento e, baseada neles, a definição de direcionamentos deliberados para o futuro. Destacase a importância da estruturação de estudos com a participação efetiva dos agentes do setor da construção, não só como respondentes de uma pesquisa, mas com real envolvimento na rotina, como na experiência empreendida por Pasquire et al (2005), que trouxe à tona a dificuldade da obtenção de informações passíveis de subsidiar de fato a tomada de decisão - questão de suma importância, que vinha sendo subestimada nas propostas teóricas.

Diante do exposto e após analisar a NBR15.575:2013, fica evidente a necessidade de estabelecer-se uma 
estrutura mais abrangente de suporte à tomada de decisão durante o desenvolvimento dos projetos. Faz-se necessário trazer para o centro do processo mais clareza a respeito das demandas, do fluxo de informação e da sustentabilidade. O conhecimento sobre a articulação em torno desta mudança de paradigma contribui para uma compreensão sistêmica, para a identificação dos desafios e para a elaboração de estratégias necessárias para operar as transformações emergentes. A NBR 15.575:2013, trata de demandas de base a serem respeitadas, mas, além de estabelecer unicamente padrões mínimos de exigência, que muitas vezes estão aquém do desejável, eles têm um escopo limitado, que não contempla questões cruciais de uma forma mais abrangente - como a interação dos aspectos tecnológicos com aqueles relacionados à concepção de projeto, a avaliação das respostas humanas, a gestão adequada do empreendimento ou a contribuição para vários dos aspectos da sustentabilidade ou do desenvolvimento. Para que a qualidade do ambiente construído possa ser aprimorada, cada artífice envolvido no processo de projeto e construção deve assimilar o conhecimento trazido pelos demais, buscando uma articulação de todas as perspectivas, para que se possa chegar a uma visão sistêmica e definir prioridades entre demandas que, muitas vezes, podem ser conflitantes.

\section{Considerações finais}

Este artigo examinou a literatura no que se refere à importância da definição de atributos para as tomadas de decisão de projeto que permitam uma gestão integrada, segundo pontos de vista ampliados comparados à abordagem da NBR15.575:2013.

No âmbito mundial, o questionamento sobre os requisitos está em curso, mas ainda não foi sistematizado, e no Brasil, ainda é incipiente. Porém, já é possível e necessário articular um diálogo entre os diversos tomadores de decisão que atuam no projeto e na construção de habitações. Considerando a característica multicritério do tema, há disponíveis diversas metodologias de tomada de decisão para dar suporte a esse processo. O estabelecimento de requisitos e critérios conjuntamente deverá contribuir para uma visão mais completa do termo “desempenho".
Além da dificuldade inerente à promoção de uma discussão deste nível, tal processo vai naturalmente gerar custos adicionais para o empreendimento. Desta maneira, a ampliação das exigências de desempenho para outros campos provavelmente não será tarefa fácil e a discussão pode ficar limitada aos detentores de recursos financeiros. Seria muito relevante haver um organismo de controle para conduzir esse processo. Neste sentido, depreende-se a importância do desenvolvimento de políticas públicas que regulem a questão. O problema já foi identificado e é necessário dar continuidade ao processo e estabelecer uma agenda de discussão que envolva todos os atores atuantes na construção, inclusive instituições capazes de representar as necessidades subjacentes da sociedade.

\section{Referências}

BERNARDES, A. A; SILVA, A. R; TAVARES, R.T; LOURA, R. M; SIRQUEIRA, C. A. S. NBR15.575:2013: Vida útil de projeto. In HABITAR 2014, Anais... Nova Lima, 2014.

CHEN, Y. et al. Sustainable performance criteria for construction method selection in concrete buildings. Automation in Construction. v. 19, n. 2, p. 235-244, Mar. 2010.

CRONNBERG, T. et al, On structuring performance requirements for buildings. In: PERFOMANCE CONCEPT IN BUILDINGS SYMPOSIUM, 1. May 2-5, 1972, Philadelphia. Proceedings..., Philadelphia: RILEM/ ASTM/CIB, 1972b. p. 23 - 30. Vol. 1.

CRONNBERG, T. et al. Performance requirements for buildings. In: PERFOMANCE CONCEPT IN BUILDINGS SYMPOSIUM, 1. May 2-5, 1972a, Philadelphia. Proceedings..., Philadelphia: RILEM/ ASTM/CIB, 1972a. p. 13 a 22. Vol. 1.

FINGER, H. The role of the performance Concept in Operation Breakthrough. In: PERFOMANCE CONCEPT IN BUILDINGS SYMPOSIUM, 1. May 2-5, 1972, Philadelphia. Proceedings..., Philadelphia: RILEM/ ASTM/CIB, 1972. p. 819 839. Vol. 2.

FOSTER, B. (Ed.). Abstract. In: PERFOMANCE CONCEPT IN BUILDINGS SYMPOSIUM, 1. May 2-5, 1972, Philadelphia. Proceedings..., Philadelphia: RILEM/ ASTM/CIB, 1972. p. xvi. Vol. 2. 
GROSS, J.G. 1996. Developments in the application of the performance concept in building. In: CIBAASTM-ISO-RILEM INTERNATIONAL SYMPOSIUM, 3, 1996, Tel Aviv. Proceedings..., Tel Aviv: Becker. R. and Paciuk, M. (Eds.), 1996. [s.p.] Vol. 1, I- 1. Disponível em: http://fire.nist.gov/bfrlpubs/build96/PDF/b96145.p df. Acesso em: 10 Dez 2014

HYUN, C.T. Making value engineering as an effective tool for Project integration using constructability and partnering concepts. SAVE INTERNATIONAL CONFERENCE, 1, Proceedings.... 1997. p.111-118. Disponível em: http://www.value-

eng.org/pdf docs/conference proceedings/1997/97 16.PDF. Acesso em: 22 Nov. 2015

LEMER, A. C.; MOAVENZADEH, F. Performance of systems of constructed facilities. In: PERFOMANCE CONCEPT IN BUILDINGS SYMPOSIUM, 1. May 2-5, 1972, Philadelphia. Proceedings..., Philadelphia: RILEM/ ASTM/CIB, 1972. p. 63 - 72. Vol. 1.

MANN, T., BENDER. Application of unobtrusive observation techniques in building performance, In: PERFOMANCE CONCEPT IN BUILDINGS SYMPOSIUM, 1. May 2-5, 1972, Philadelphia. Proceedings..., Philadelphia: RILEM/ ASTM/CIB, 1972. p. 93-100, Vol. 1.

MARTHUR, K.; McGEORGE, D. An integrated decision making environment for cost vs quality control. In: BEZELGA, A.; BRANDON, P.S. (Ed.) Management, Quality and Economics in Building. Taylor and Francis: London; 2006. p. 692 - 701.

PASQUIRE, C. et al. What Should You Really Measure if You Want to Compare Prefabrication With Traditional Construction? In: ANNUAL CONFERENCE OF THE INTERNATIONAL GROUP FOR LEAN CONSTRUCTION, 13.2005. Sydney, Australia. Proceedings..., Sidney, 2005. p. 481-491

PENA, W. M.; FOCKE, J. W. Performance requirements of buildings and the whole problem. In: PERFOMANCE CONCEPT IN BUILDINGS SYMPOSIUM, 1. May 2-5, 1972, Philadelphia. Proceedings..., Philadelphia: RILEM/ ASTM/CIB, 1972. p. 43 - 56. Vol. 1.

PICANÇO, E. [199?]. Processo Decisório. Rio de Janeiro, Universidade Federal Fluminense. apostila, $72 \mathrm{p}$.
ROY, B. Multicriteria methodology for decisions Aiding. The Netherlands: Kluwer Academic Publishers, 1998.

SILVA, M. A. C. Metodologia de seleção tecnológica na produção de edificações com o emprego do conceito de custos ao longo da vida útil. 1996. $356 \mathrm{f}$. Tese (Doutorado em Engenharia Civil) - Escola Politécnica, Universidade de São Paulo, São Paulo.

STAATS, B. B. Operation Breakthrough: Lessons Learned about Demonstrating New Technology. Report to the Congress. Washington D.C. November 2, 1976. 87 p. Disponível em: http://www.gao.gov/assets/120/117465.pdf. Acesso em: 03 fev 2015

UGWU, O. O., HAUPT, T. C. Building and Environment. Key performance indicators and assessment methods for infrastructure sustainability - a South African construction industry perspective. Building and Environment. v.42. n.2, 2007. p.665680.

UNEP - United Nations Environment Programme. AGENDA 21. Integrating Environment and Development in Decision-Making. (Ch. 8) UNITED NATIONS CONFERENCE ON ENVIRONMENT AND DEVELOPMENT. Rio de Janeiro, Brazil, 3 to 14 June. 1992, Disponível em: http://www.unep.org/Documents.Multilingual/Def ault.asp? DocumentID=52\&ArticleID=56 Acesso em: 12 set. 2015

UTOMO, C.; IDRUS, A. A Concept toward Negotiation Support for Value Management on Sustainable Construction. Journal of Sustainable Development. v. 4, n. 6, 2011. p 56- 66 - Disponível em:

http://www.ccsenet.org/journal/index.php/jsd/artic le/view/8643; Acesso: 21 jan 2015.

VAN DER HEIJDEN, K.;_Can internally generated futures accelerate organizational learning? Futures, Mar 2004, v. 36 n. 2, p.145-159.

YUNUS, R., YANG, J. An integrated approach to enhance sustainability in industrialized building systems. In: ASIA PACIFIC STRUCTURAL ENGINEERING \& CONSTRUCTION CONFERENCE, 8. 2012, Surabaya. Proceedings... Surabaya: MD. NOOR, NORHAZILAN \& ISMAIL, MOHAMMAD (Eds.), 2012. [s.p.] Disponível em: http://eprints.qut.edu.au/54731/Acesso em: 07 Nov. 2014. 